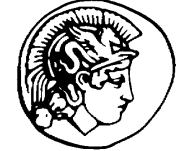

PERGAMON
Science@Directo

International Journal of Non-Linear Mechanics 38 (2003) 1561-1579
NON-LINEAR

MECHANICS

www.elsevier.com/locate/ijnonlinmec

\title{
Linear and non-linear analyses of convection in a micropolar fluid occupying a porous medium
}

\author{
P.G. Siddheshwar ${ }^{a}, *$ C.V. Sri Krishna ${ }^{b}$ \\ ${ }^{a}$ Department of Mathematics, Bangalore University, Central College Campus, Bangalore 560 001, India \\ ${ }^{\mathrm{b}}$ Department of Mathematics, Sir M. Visvesvaraya Institute of Technology, Bangalore 562 157, India
}

Received 12 October 2001; accepted 25 June 2002

\begin{abstract}
Linear and weakly non-linear analyses of convection in a micropolar fluid occupying a high-porosity medium are performed. The Brinkman-Eringen momentum equation is considered. The linear and non-linear analyses are, respectively, based on the normal mode technique and truncated representation of Fourier series. The linear theory for a two-phase system reiterates that the preferred mode of convection is stationary as in the case of a single-phase system. An autonomous system of differential equations representing cellular convection arising in the study is considered to analyse the critical points. The Nusselt number is obtained as a function of micropolar and porous medium parameters.
\end{abstract}

(C) 2002 Elsevier Science Ltd. All rights reserved.

Keywords: Rayleigh-Benard convection; Micropolar fluids; Porous media; Double Fourier series; Linear and non-linear analyses

\section{Introduction}

It is now common knowledge that fluids with suspended particles are a working medium in many an industrial application. The fluids most often do not subscribe to a Newtonian description and the advent of microcontinuum field theories in such a situation threw open new fertile fields of application (see [1-4]). The common comment in respect of micropolar fluids was that there are no experiments whatsoever in which any of the material moduli could be measured. The work of Migoun [5] demonstrated an experimental method of determining parameters characterizing the microstructure of such fluids and seems to have laid to rest many doubts on the theory.

The theory of thermomicropolar convection began with Datta and Sastry [6] and interestingly continued by Ahmadi [7], Lebon and Perez Garcia [8], Bhattacharya and Jena [9], and Payne and Straughan [10]. The above works give a good understanding of Rayleigh-Benard convection in micropolar fluids. The study of natural convection in micropolar fluids in the presence of external constraints has been also investigated. Qin and Kaloni [11] have discussed the thermal instability in rotating micropolar fluids and established that the non-inertial acceleration is a possible means of controlling convection. Recently Siddheshwar and Pranesh

\footnotetext{
${ }^{*}$ Corresponding author. Tel.: +91-80-2220483; fax: +91-80-2259843.

E-mail addresses: pgsiddheshwar@hotmail.com (P.G. Siddheshwar), cvsrikrishna@hotmail.com (C.V. Sri Krishna).
} 
[12-17] and Siddheshwar and Dessaux [18] have shown that the magnetic field, non-uniform basic temperature/salinity and thermal/gravity modulation are also effective mechanisms of controlling convection. All the above investigations mentioned earlier have been addressed for a purely viscous case, i.e., no porous matrix.

Investigations related to convection in micropolar fluids occupying a porous medium are sparse. Sharma and Gupta [19] have studied the thermal convection in micropolar fluids in a porous medium. The effect of rotation on thermal convection in micropolar fluids occupying a porous medium has been studied by Sharma and Kumar [20]. El-Hakiem [21] has investigated the effect of transverse magnetic field on natural convection in a boundary layer flow field as well as in the porous medium.

The aforementioned works concern linear theory and a good understanding of non-linear convection is warranted in this type of practically important fluids to assess the amount of heat transfer, finite amplitude cellular motion and other related aspects. A perusal of available literature reveals that Payne and Straughan [22] have studied non-linear convection in micropolar fluids by employing the energy method and existence of sub-critical instabilities was not inferred from their work. Franchi and Straughan [23] have investigated non-linear stability for thermal convection in a micropolar fluid with temperature dependent viscosity. They infer that due to complicated non-linearities which arise, it is necessary to use a generalised energy analysis which requires an intricate use of embedding inequalities. The second-order non-linear systems arising in convective flow of a micropolar fluid has also been studied by Vajravelu et al. [24] in a porous medium.

The analog of problems in micropolar fluids reported above, does not seem to have been addressed in a porous medium. The non-linear realm of convection in micropolar fluids in a porous medium is also presently unaddressed. In view of this, the non-linear convection problem in micropolar fluids occupying a porous medium is investigated in this paper.

\section{Mathematical formulation}

Consider a horizontal porous layer of infinite extent occupied by a Boussinesquian micropolar fluid (fluid with suspended particles). We note here that the Oberbeck-Boussinesq approximation even for the classical linearly viscous fluid is a rather subtle matter. At this point of time there seems to be no reported work that addresses this issue in micropolar fluids. We take the view that a method similar to that carried out by Rajagopal et al. [25] needs to be followed in justifying the Oberbeck-Boussinesq approximation for micropolar fluids. As there does not seem to be any major factor in micropolar fluids that could contradict the OberbeckBoussinesq approximation, we assume the same in the present paper.

We assume that the fluid layer has thickness ' $d$ ' and $\Delta T$ to be the adverse temperature gradient. The fluid is assumed to be non-Newtonian and describable by Eringen [3] constitutive equation. The present investigation in a porous medium demands an appropriate theory to study two-phase systems. There are rigorous theories in place to study diffusion of fluid through a porous medium (see [26-28]). For Newtonian fluids flowing through a porous medium, equations for momentum and heat transfer have been rigorously derived and are now common knowledge (see [27]). In the case of present problem involving a fluid with microstructure flowing through a porous medium we believe that the microstructures modify the Darcy resistance just as they would the viscous shear. With this understanding and firm belief we introduce a microstructure modified Darcy friction, in addition to the viscous shear. A similar reasoning can be put forth in respect of the assumed single-phase heat transfer equation arrived at by deftly combining the laws of micropolar heat conduction and porous media heat transfer.

In the present work, we use Dupit-Forchheimer relation to give corrections to momentum and angular momentum equations to account for changes in velocity and spin and assume that the porous medium is in thermal equilibrium (i.e. temperature of fluid and temperature of solid particles in porous medium is constant). This is a reasonable assumption of porous medium to consider the general balance laws of mixtures. For 
mathematical tractability we confine ourselves to two-dimensional rolls so that all the quantities except the spin are independent of $y$, a horizontal co-ordinate. Further the boundaries are assumed to be free-free, spin-free with isothermal conditions. Thus the governing equations for a Rayleigh-Benard situation in a Boussinesquian micropolar fluid-saturated porous medium are:

$$
\begin{aligned}
& q_{i, i}=0, \\
& \left\{\rho_{0}\left[\frac{1}{\Phi} \frac{\partial q_{i}}{\partial t}+\frac{1}{\Phi^{2}} q_{j} q_{i, j}\right]+p_{, i}+\rho g \delta_{i 3}+\left(\frac{\zeta+\mu_{\mathrm{f}}}{K}\right) q_{i}\right\}=\left(\frac{2 \zeta}{\Phi}+\mu^{\prime}\right) q_{i, j j}+\frac{\zeta}{\Phi} \varepsilon_{i j k} \tilde{\omega}_{k, j}, \\
& \rho_{0} I\left[\frac{\partial \tilde{\omega}_{i}}{\partial t}+\frac{1}{\Phi} q_{j} \tilde{\omega}_{i, j}\right]=\left(\lambda^{\prime}+\eta^{\prime}\right) \tilde{\omega}_{i, i j}+\eta^{\prime} \tilde{\omega}_{i, k k}+\zeta\left(\varepsilon_{i j k} q_{k, j}-2 \tilde{\omega}_{i}\right), \\
& M \frac{\partial T}{\partial t}+q_{j} T_{, j}=\frac{\beta}{\rho_{0} C_{v} \Phi} \varepsilon_{i j k} \tilde{\omega}_{k, j} T_{, k}+\chi_{\mathrm{e}} T_{, k k}, \\
& \rho=\rho_{0}\left[1-\alpha_{t}\left(T-T_{0}\right)\right],
\end{aligned}
$$

where $q_{i}$ is the component of velocity vector, $M$ is the heat capacity ratio given by

$$
M=\frac{(1-\Phi)\left(\rho C_{\mathrm{p}}\right)_{\text {solid }}+\Phi\left(\rho C_{\mathrm{p}}\right)_{\text {fluid }}}{\left(\rho C_{\mathrm{p}}\right)_{\text {fluid }}} .
$$

The effective thermal diffusivity $\chi_{\mathrm{e}}$ is given by

$$
\chi_{\mathrm{e}}=\frac{\Phi \chi_{\text {fluid }}+(1-\Phi) \chi_{\text {solid }}}{\left(\rho C_{\mathrm{p}}\right)_{\text {fluid }}},
$$

where $\Phi$ is the porosity, $\mu_{\mathrm{f}}$ is the viscosity of the fluid, $\mu^{\prime}$ is the effective viscosity of the porous medium, $\zeta$ is the coupling viscosity, $\eta^{\prime}$ is the bulk viscosity co-efficient, $\lambda^{\prime}$ is the shear spin viscosity co-efficient, $\beta$ is the micropolar heat conduction co-efficient, $\tilde{\omega}$ is the spin and $C_{\mathrm{p}}$ is the specific heat at constant pressure. In Eq. (2) $K$ is the permeability and is related to $\Phi$ by Karman-Kozeny relation:

$$
K=\frac{\mathrm{d}_{\mathrm{p}}^{2} \Phi^{2}}{150(1-\Phi)^{2}},
$$

where $d_{\mathrm{p}}$ is the diameter of spherical particles making up the porous medium. Other quantities in Eqs. (1)-(5) have conventional meaning (see [29]).

\section{Basic state}

The basic state of the fluid is quiescent and is described by

$$
\begin{aligned}
& q_{i \mathrm{~b}}=(0,0,0), \quad \tilde{\omega}_{i \mathrm{~b}}=(0,0,0), \\
& T=T_{\mathrm{b}}(z), \quad \rho=\rho_{\mathrm{b}}(z), \quad p=p_{\mathrm{b}}(z),
\end{aligned}
$$

where the subscript $\mathrm{b}$ denotes quantities in the basic state. The basic state pressure $p_{\mathrm{b}}$, temperature $T_{\mathrm{b}}$ and density $\rho_{\mathrm{b}}$ satisfy the equations

$$
\begin{aligned}
& \frac{\mathrm{d} p_{\mathrm{b}}}{\mathrm{d} z}+\rho_{\mathrm{b}} g=0, \\
& \frac{\mathrm{d}^{2} T_{\mathrm{b}}}{\mathrm{d} z^{2}}=0
\end{aligned}
$$


and

$$
\rho_{\mathrm{b}}=\rho_{0}\left[1-\alpha_{t}\left(T_{\mathrm{b}}-T_{0}\right)\right] .
$$

Eqs. (1) and (3) are also satisfied by the basic state. The basic state of the fluid is perturbed and hence we assume

$$
\begin{array}{ll}
q_{i}=q_{i \mathrm{~b}}+q_{i}^{\prime}, & \tilde{\omega}_{i}=\tilde{\omega}_{i \mathrm{~b}}+\tilde{\omega}_{i}^{\prime}, \\
T=T_{\mathrm{b}}+T^{\prime}, & \rho=\rho_{\mathrm{b}}+\rho^{\prime}, \quad p=p_{\mathrm{b}}+p^{\prime},
\end{array}
$$

thereby leading to the following perturbed equations:

$$
\begin{aligned}
& \left\{\rho_{0}\left[\frac{1}{\Phi} \frac{\partial q_{i}}{\partial t}+\frac{1}{\Phi^{2}} q_{j}^{\prime} q_{i, j}^{\prime}\right]+p_{, i}^{\prime}+\rho^{\prime} g \delta_{i 3}+\left(\frac{\zeta+\mu_{\mathrm{f}}}{K}\right) q_{i}^{\prime}\right\}=\left(\frac{2 \zeta}{\Phi}+\mu^{\prime}\right) q_{i, j j}^{\prime}+\frac{\zeta}{\Phi} \varepsilon_{i j k} \tilde{\omega}_{k, j}^{\prime}, \\
& \rho_{0} I\left[\frac{\partial \tilde{\omega}_{i}^{\prime}}{\partial t}+\frac{1}{\Phi} q_{j}^{\prime} \tilde{\omega}_{i, j}^{\prime}\right]=\left(\lambda^{\prime}+\eta^{\prime}\right) \tilde{\omega}_{i, j}^{\prime}+\eta^{\prime} \tilde{\omega}_{i, j j}^{\prime}+\zeta\left[\varepsilon_{i j k} q_{k, j}^{\prime}-2 \tilde{\omega}_{i}^{\prime}\right], \\
& M \frac{\partial T^{\prime}}{\partial t}+q_{j}^{\prime} T_{, j}^{\prime}=\frac{\beta}{\rho_{0} C_{v} \Phi} \varepsilon_{i j k} \tilde{\omega}_{k, j}^{\prime}+\chi_{\mathrm{e}} T_{, j j}^{\prime} \\
& \rho^{\prime}=-\alpha_{t} \rho_{0} T^{\prime},
\end{aligned}
$$

where the prime for $q, \omega, T, p$ and $\rho$ denotes quantities of the perturbed state.

We now non-dimensionalise the above equations using the following definitions:

$$
\begin{aligned}
& \left(x^{*}, y^{*}, z^{*},\right)=\left(\frac{x}{d}, \frac{y}{d}, \frac{z}{d}\right), \quad t^{*}=\left(\frac{t}{M d^{2} / \chi_{\mathrm{e}}}\right), \\
& q_{i}^{*}=\frac{q_{i}^{\prime}}{\left(\chi_{\mathrm{e}} / d\right)}, \quad \Omega=\frac{\left(\varepsilon_{i j k} \tilde{\omega}_{k, j}^{\prime}\right)_{z}}{\chi_{\mathrm{e}} / d^{3}}, \\
& T^{*}=\frac{T^{\prime}}{\Delta T}, \quad \tilde{\omega}_{i}^{*}=\frac{\tilde{\omega}_{i}^{\prime}}{\chi_{\mathrm{e}} / d^{2}} \quad \text { and } \\
& p^{*}=\frac{p^{\prime}}{\chi_{\mathrm{e}}\left(\frac{\zeta+\mu_{\mathrm{f}}}{d^{2}}\right)} .
\end{aligned}
$$

Using (14) in Eqs. (10)-(12), we get

$$
\begin{aligned}
& \frac{\lambda}{\operatorname{Pr}} \frac{\partial q_{i}}{\partial t}+\frac{1}{\operatorname{Pr}} q_{j} q_{i, j}+p_{, i}+D a q_{i}=-R_{\mathrm{m}} T \delta_{i 3}+\left(N_{1}+N_{1}^{\prime}\right) q_{i, j j}+N_{1} \varepsilon_{i j k} \tilde{\omega}_{k, j}, \\
& \frac{\lambda N_{2}}{\operatorname{Pr}} \frac{\partial \tilde{\omega}_{i}}{\partial t}+\frac{N_{2}}{\operatorname{Pr}} q_{j} \tilde{\omega}_{i, j}=N_{4} \tilde{\omega}_{i, i j}+N_{3} \tilde{\omega}_{i, j j}+N_{1}\left[\varepsilon_{i j k} q_{k, j}-2 \tilde{\omega}_{i}\right], \\
& \frac{\partial T}{\partial t}+q_{i}-N_{5} \varepsilon_{i j k} \tilde{\omega}_{k, j}=T_{, k k},
\end{aligned}
$$

where the asterisks have been dropped for simplicity and use has been made of Eq. (13) in Eq. (10). 
The non-dimensional quantities appearing in Eqs. (15)-(17) are

$$
\begin{aligned}
& \lambda=\frac{\Phi}{M}, \\
& N_{1}=\frac{\zeta}{\Phi\left(\mu_{\mathrm{f}}+\zeta\right)} \quad \text { (Coupling parameter), } \\
& N_{2}=\frac{I}{d^{2}} \quad(\text { Microinertia parameter }), \\
& N_{3}=\frac{\eta^{\prime}}{d^{2}\left(\mu_{\mathrm{f}}+\zeta\right) \Phi} \quad \text { (Couple stress parameter), } \\
& N_{5}=\frac{\beta}{\Phi \rho_{0} C_{v} d^{2}} \quad(\text { Micropolar heat conduction parameter }), \\
& \operatorname{Pr}=\frac{\left(\zeta+\mu_{\mathrm{f}}\right) \Phi^{2}}{\rho_{0} \chi_{\mathrm{e}}} \quad \text { (Effective Prandtl number), } \\
& N_{1}^{\prime}=\frac{\mu^{\prime}+\zeta / \Phi}{\left(\mu_{\mathrm{f}}\right)} \quad \text { (Modified viscosity ratio), } \\
& D a=\frac{d^{2}}{k} \quad(\text { Inverse Darcy number), } \\
& R_{\mathrm{m}}=\frac{\rho_{0} \alpha g \Delta T d^{3}}{\left(\mu_{\mathrm{f}}+\zeta\right) \chi_{\mathrm{e}}} \quad \text { (Effective micropolar Rayleigh number). }
\end{aligned}
$$

We note here that for $\zeta=0$, we get the classical non-dimensional parameters associated with Newtonian fluids occupying a porous medium.

Operating curl on the linear momentum transport Eq. (15) and on the angular momentum Eq. (16) we get the following non-dimensional form of vorticity, microrotation and the heat transport:

$$
\begin{aligned}
& \left(\frac{\lambda}{\operatorname{Pr}} \frac{\partial}{\partial t}+D a\right) \nabla^{2} \psi=-R_{\mathrm{m}} \frac{\partial T}{\partial x}+\left(N_{1}+N_{1}^{\prime}\right) \nabla^{4} \psi-N_{1} \nabla^{2} \tilde{\omega}_{y}+\frac{\lambda}{\operatorname{Pr}} J\left(\psi, \nabla^{2} \psi\right), \\
& \frac{\lambda N_{2}}{\operatorname{Pr}} \frac{\partial \tilde{\omega}_{y}}{\partial t}=N_{3} \nabla^{2} \tilde{\omega}_{y}=N_{1} \nabla^{2} \psi-2 N_{1} \tilde{\omega}_{y}+\frac{N_{2}}{\operatorname{Pr}} J\left(\psi, \tilde{\omega}_{y}\right), \\
& \frac{\partial T}{\partial t}=\frac{\partial \psi}{\partial x}+\nabla^{2} T-N_{5} \frac{\partial \tilde{\omega}_{y}}{\partial x}+N_{5} J\left(\tilde{\omega}_{y}, T\right)+J(\psi, T),
\end{aligned}
$$

where $\psi$ is the stream function defined as

$$
u=\frac{\partial \psi}{\partial z}, \quad w=-\frac{\partial \psi}{\partial x} .
$$

Eqs. (18)-(20) are solved for stress-free, isothermal, spin-free boundaries and hence the boundary conditions are

$$
\psi=\frac{\partial^{2} \psi}{\partial z^{2}}=T=\tilde{\omega}_{y}=0 \quad \text { at } z=0,1
$$

In what follows we now discuss the linear theory. 


\section{Linear stability analysis}

To study linear theory we use the linearised version of (18)-(20) and seek the solutions to the linearised equations in the form (see [29])

$$
\left[\begin{array}{c}
\psi \\
\tilde{\omega}_{y} \\
T
\end{array}\right]=\mathrm{e}^{\mathrm{i} \omega_{1} t}\left[\begin{array}{c}
A \sin \pi \alpha x \\
B \sin \pi \alpha x \\
C \cos \pi \alpha x
\end{array}\right] \sin \pi z
$$

which satisfy (22). Substituting (23) into Eqs. (18)-(20), we get a system of three homogeneous equations for $A, B$ and $C$. For a non-trivial solution to $A, B$ and $C$, we require

$$
R_{\mathrm{m}}=\frac{\left[\frac{N_{2} \lambda \mathrm{i} \omega_{1}}{P r}+\left(2 N_{1}+N_{3} \delta^{2}\right)\right]\left\{\frac{\lambda \mathrm{i} \omega_{1}}{P r}+\left[D a+\left(N_{1}+N_{1}^{\prime}\right) \delta^{2}\right] \delta^{2}-N_{1}^{2} \delta^{4}\right\}\left(\mathrm{i} \omega_{1}+\delta^{2}\right)}{(\pi \alpha)^{2}\left[\frac{N_{2} \lambda \mathrm{i} \omega_{1}}{P r}+\left(2 N_{1}+N_{3} \delta^{2}\right)-N_{1} N_{5} \delta^{2}\right]} .
$$

By separating the real and imaginary parts in Eq. (24), we get

$$
R_{\mathrm{m}}=\frac{K_{5}\left\{K_{10}\left(K_{6}-\omega_{1}^{2} K_{8}\right)+\omega_{1}^{2} K_{3}\left(K_{7}-\omega_{1}^{2} K_{9}\right)\right\}}{\left(K_{10}^{2}+\omega_{1}^{2} K_{3}^{2}\right)}+\mathrm{i} \omega_{1} N
$$

where

$$
\begin{aligned}
& N=K_{5}\left\{\frac{K_{10}\left\{\left(K_{7}-\omega_{1}^{2} K_{9}\right)+K_{3}\left(K_{6}-\omega_{1}^{2} K_{8}\right)\right\}}{\left(K_{10}^{2}+\omega_{1}^{2} K_{3}^{2}\right)}\right\}, \\
& K_{1}=\frac{\lambda}{\operatorname{Pr}}, \quad K_{2}=\left[D a \delta^{2}+\left(N+N_{1}^{\prime}\right) \delta^{4}\right], \\
& \delta^{2}=\pi^{2}\left(1+\alpha^{2}\right), \quad \alpha^{2}=\frac{a^{2}}{\pi^{2}}, \\
& K_{3}=N_{2} K_{1}, \quad K_{4}=2 N_{1}+N_{3} \delta^{2}, \\
& K_{5}=\frac{\delta^{2}}{\pi^{2} \alpha^{2}}, \quad K_{6}=\left(K_{2} K_{4}-N_{1}^{2} \delta^{4}\right), \\
& K_{7}=\left[\left(K_{2} K_{4}-N_{1}^{2} \delta^{4}\right)+\delta^{4}\left(K_{1} K_{4}+K_{2} K_{3}\right)\right], \\
& K_{8}=\left[K_{1} K_{4}+\delta^{2}\left(K_{1} K_{4}+K_{2} K_{3}\right)\right], \\
& K_{9}=\frac{\lambda}{\operatorname{Pr}}\left(2 N_{1}+N_{3} \delta^{2}\right),
\end{aligned}
$$

and

$$
K_{10}=\left[\left(2 N_{1}+N_{3} \delta^{2}\right)-N_{1} N_{5} \delta^{2}\right] .
$$

In view of the observation on the 'Principle of Exchange of Stability' made by Lebon and Perez Garcia [8] and Siddheshwar and Pranesh $[11,12]$ we consider only stationary convection, i.e. $\omega_{1}=0$. Thus the expression 
Table 1

Comparison of critical Rayleigh number and critical wave number between Newtonian and Micropolar for different values of $D a$

\begin{tabular}{lllll}
\hline$D a$ & \multicolumn{2}{l}{ Newtonian } & & \multicolumn{2}{l}{ Micropolar } \\
\cline { 2 - 5 } & $R_{\mathrm{c}}$ & $a_{\mathrm{c}}^{2}$ & $R_{\mathrm{mc}}$ & $a_{\mathrm{c}}^{2}$ \\
\hline 1.0 & 642.60 & 5.10 & 1156.54 & 5.00 \\
5.0 & 816.69 & 5.70 & 1340.67 & 5.40 \\
10.0 & 1028.73 & 6.20 & 1566.41 & 6.80 \\
15.0 & 1238.19 & 6.60 & 1788.72 & 6.10 \\
20.0 & 1443.45 & 6.90 & 2008.57 & 6.30 \\
\hline
\end{tabular}

Chosen parameter values: $N_{1}^{\prime}=1.5, N_{1}=0.10, N_{3}=2.0, N_{5}=1.0$. Remarks: (i) $R_{\mathrm{c}}<R_{\mathrm{mc}},\left(a_{\mathrm{c}}^{2}\right)_{\text {Micropolar }}<\left(a_{\mathrm{c}}^{2}\right)_{\text {Newtonian. }}$ (ii) The effect of $D a$ on $R_{\mathrm{mc}}$ is qualitatively similar to that of the Newtonian problem in a porous medium.

Table 2

Comparison of the effect of viscosity ratio (Brinkman number) on the critical Rayleigh number and critical wave number for Newtonian and Micropolar fluids

\begin{tabular}{lllll}
\hline Viscosity ratio & \multicolumn{2}{l}{ Newtonian } & \multicolumn{2}{l}{ Micropolar } \\
\cline { 2 - 5 }$\left(N_{1}^{\prime}\right)$ & $R_{\mathrm{c}}$ & $a_{\mathrm{c}}^{2}$ & $R_{\mathrm{mc}}$ & $a_{\mathrm{c}}^{2}$ \\
\hline 0.80 & 749.75 & 5.80 & 852.97 & 5.70 \\
0.90 & 816.69 & 5.70 & 922.78 & 5.60 \\
1.00 & 883.58 & 5.60 & 992.53 & 5.60 \\
1.50 & 1217.22 & 5.40 & 1340.67 & 5.40 \\
2.00 & 1550.25 & 5.30 & 1688.29 & 5.20 \\
2.50 & 1883.00 & 5.30 & 2035.68 & \\
\hline
\end{tabular}

Chosen parameter values: $D a=5.0, N_{1}=0.1, N_{3}=2.0, N_{5}=1.0$. Remarks: (i) The effect of $N_{1}^{\prime}$ on $R_{\mathrm{mc}}$ is qualitatively similar to that of the effect of ratio of viscosities, $\Lambda$, for Newtonian problem in a porous medium. (ii) $R_{\mathrm{c}}<R_{\mathrm{mc}}$, $\left(a_{\mathrm{c}}^{2}\right)_{\text {Micropolar }}<\left(a_{\mathrm{c}}^{2}\right)_{\text {Newtonian }}$.

Table 3

Comparison of critical Rayleigh number, $R_{\mathrm{mc}}$, and critical wave number, $a_{\mathrm{c}}^{2}$, for different values coupling parameter, $N_{1}$

\begin{tabular}{lllll}
\hline$N_{1}$ & \multicolumn{2}{l}{ Porous case } & \multicolumn{2}{c}{ Purely viscous case [30] } \\
\cline { 2 - 5 } & $R_{\mathrm{mc}}$ & $a_{\mathrm{c}}^{2}$ & $R_{\mathrm{mc}}$ & $a_{\mathrm{c}}^{2}$ \\
\hline 0.00 & 1204.85 & 5.42 & 657.51 & 4.94 \\
0.01 & 1217.85 & 5.42 & 667.42 & 4.93 \\
0.05 & 1271.11 & 5.41 & 707.98 & 4.93 \\
0.10 & 1340.67 & 5.39 & 760.83 & 4.93 \\
0.20 & 1490.69 & 5.36 & 874.42 & 4.93 \\
0.50 & 2051.47 & 5.27 & 1293.95 & 4.90 \\
0.80 & 2862.55 & 5.16 & 1885.35 & 4.83 \\
1.00 & 3636.41 & 5.05 & 2433.42 & 4.77
\end{tabular}

Chosen parameter values: (i) Viscous case $D a=0, N_{1}^{\prime}=1.0, N_{3}=2.0, N_{5}=1.0$. (ii) Porous case $D a=5.0, N_{1}^{\prime}=1.5, N_{3}=2.0, N_{5}=1.0$. Remarks: (i) $\left(R_{\mathrm{mc}}, a_{\mathrm{c}}^{2}\right)_{\text {viscous }}<\left(R_{\mathrm{mc}}, a_{\mathrm{c}}^{2}\right)_{\text {porous }}$. (ii) $R_{\mathrm{mc}}$ increases with $N_{1}$.

to find the stationary critical effective micropolar Rayleigh number, $R_{\mathrm{mc}}$ corresponding to the critical wave number, $a_{\mathrm{c}}^{2}$ is given by

$$
R_{\mathrm{mc}}=\frac{\left\{\delta^{2}\left[D a+\left(N_{1}+N_{1}^{\prime}\right) \delta^{2}\right]\left(2 N_{1}+N_{3} \delta^{2}\right) N_{1}^{2} \delta^{4}\right\} \delta^{2}}{(\pi \alpha)^{2}\left\{\left(2 N_{1}+N_{3} \delta^{2}\right)-N_{1} N_{5} \delta^{2}\right\}} .
$$


Table 4

Comparison of $R_{\mathrm{mc}}$ and $a_{\mathrm{c}}^{2}$ for different couple stress parameter, $N_{3}$

\begin{tabular}{lllll}
\hline$N_{3}$ & \multicolumn{2}{l}{ Porous case } & \multicolumn{2}{l}{ Purely viscous case [30] } \\
\cline { 2 - 5 } & $R_{\mathrm{mc}}$ & $a_{\mathrm{c}}^{2}$ & $R_{\mathrm{mc}}$ & $a_{\mathrm{c}}^{2}$ \\
\hline 0.50 & 1597.68 & 5.40 & 897.10 & 4.92 \\
1.00 & 1417.64 & 5.40 & 801.96 & 4.93 \\
1.50 & 1365.49 & 5.40 & 774.12 & 4.93 \\
2.00 & 1340.67 & 5.40 & 760.83 & 4.93 \\
2.50 & 1326.16 & 5.40 & 753.05 & 4.93 \\
3.00 & 1316.64 & 5.40 & 747.94 & 4.93 \\
5.00 & 1297.96 & 5.40 & 737.90 & 4.93 \\
8.00 & 1287.65 & 5.40 & 732.35 & 4.93 \\
\hline
\end{tabular}

Chosen parameter values: (i) Purely viscous case $D a=0, N_{1}^{\prime}=1.0, N_{1}=0.1, N_{5}=1.0$. (ii) Porous case $D a=5.0, N_{1}^{\prime}=1.5, N_{1}=0.1$, $N_{5}=1.0$. Remarks: (i) $\left(R_{\mathrm{mc}}, a_{\mathrm{c}}^{2}\right)_{\text {viscous }}<\left(R_{\mathrm{mc}}, a_{\mathrm{c}}^{2}\right)$ porous. (ii) $R_{\mathrm{mc}}$ decreases with increase in $N_{3}$.

Table 5

Comparison of $R_{\mathrm{mc}}$ and $a_{\mathrm{c}}^{2}$ for different micropolar heat conduction parameter, $N_{5}$ and different phases

\begin{tabular}{lllll}
\hline$N_{5}$ & \multicolumn{3}{l}{ Porous case } & \multicolumn{2}{c}{ Purely viscous case [30] } \\
\cline { 2 - 5 } & $R_{\mathrm{mc}}$ & $a_{\mathrm{c}}^{2}$ & $R_{\mathrm{mc}}$ & $a_{\mathrm{c}}^{2}$ \\
\hline 0.10 & 1275.90 & 5.40 & 726.66 & 4.93 \\
0.30 & 1290.81 & 5.40 & 733.98 & 4.93 \\
0.50 & 1304.68 & 5.40 & 741.46 & 4.93 \\
0.80 & 1326.04 & 5.40 & 752.96 & 4.93 \\
1.00 & 1340.67 & 5.40 & 760.83 & 4.93 \\
2.00 & 1418.96 & 5.40 & 802.79 & 4.93 \\
\hline
\end{tabular}

Chosen parameter values: (i) Purely viscous case $D a=0, N_{1}^{\prime}=1.0, N_{1}=0.1, N_{3}=2.0$. (ii) Porous case $D a=5.0, N_{1}^{\prime}=1.5, N_{1}=0.1$, $N_{3}=2.0$. Remarks: (i) $\left(R_{\mathrm{mc}}, a_{\mathrm{c}}^{2}\right)_{\text {viscous }}<\left(R_{\mathrm{mc}}, a_{\mathrm{c}}^{2}\right)_{\text {porous }}$. (ii) $R_{\mathrm{mc}}$ increases with $N_{5}$.

\subsection{Limiting cases}

The eigenvalue expression (26) is quite general. The results of viscous flow (i.e., non-porous) [30] and Darcy flow regimes can be obtained from it in the limit $D a \rightarrow 0$ (with $N_{1}^{\prime}=1$ ) and $D a \rightarrow \infty$, respectively. The effect of micropolar and porous medium parameters on $R_{\mathrm{m}}$ is shown in Tables $1-5$. In the next section we discuss the non-linear theory.

\section{Non-linear theory}

The linear theory discussed in the previous section predicts only the condition for the onset of convection and is silent about the heat transfer. To know the amount of heat transfer we have to resort to the non-linear theory. We now follow Veronis [31] and undertake a weak non-linear analysis of the problem. Although the relationship between the solutions of the governing partial differential and the corresponding severely truncated ordinary differential system has not been established these low-order spectral modes may reproduce qualitatively the convective phenomenon observed in the full system. The result can be used as starting values while discussing the fully non-linear problem. 
The first effect of non-linearity is to distort the temperature field through the interaction of $\psi$ and $T$ and $\tilde{\omega}_{y}$ and $T$. The distortion of temperature field will correspond to a change in the horizontal mean, i.e., a component of the form $\sin 2 \pi z$ will be generated. Thus the minimal system which describes finite amplitude convection in a micropolar fluid-saturated porous layer is given by

$$
\left[\begin{array}{c}
\psi \\
\tilde{\omega}_{y} \\
T
\end{array}\right]=\left[\begin{array}{c}
A(t) \sin \pi \alpha x \\
B(t) \sin \pi \alpha x \\
C(t) \cos \pi \alpha x
\end{array}\right] \sin \pi z+\left[\begin{array}{c}
0 \\
0 \\
D(t)
\end{array}\right] \sin 2 \pi z
$$

where the amplitudes $A, B, C$ and $D$ are to be determined from the dynamics of the system. The stream function and the spin do not contain an $x$-independent term because the spontaneous generation of large-scale flow has been discounted.

Substituting Eqs. (26) and first two relations of (27) into Eqs. (18)-(20), we get

$$
\begin{aligned}
& {\left[\begin{array}{c}
\dot{A} \\
\dot{B} \\
\dot{C} \\
\dot{D}
\end{array}\right]=\left[\begin{array}{cccc}
-\frac{P r}{\lambda}\left(D a \delta^{2}+\left(N_{1}^{\prime}+N_{1}\right) \delta^{4}\right) & -\frac{N_{1} \lambda}{P r} & -\frac{\pi \alpha R_{\mathrm{m}} P r}{\lambda} & 0 \\
-\frac{N_{1}}{N_{2}} \frac{\delta^{2} P r}{\lambda^{2}} & -\left(2 N_{1}+N_{3} \delta^{2}\right) \frac{P r}{N_{2} \lambda} & 0 & 0 \\
-\pi \alpha & -N_{5} \pi \alpha & -\delta^{2} & 0 \\
0 & 0 & 0 & -4 \pi^{2}
\end{array}\right]\left[\begin{array}{c}
A \\
B \\
C \\
D
\end{array}\right]} \\
& -\pi^{2} \alpha D\left[\begin{array}{c}
0 \\
0 \\
\left(A+N_{5} B\right) \\
0
\end{array}\right]+\frac{\pi^{2} \alpha C}{2}\left[\begin{array}{c}
0 \\
0 \\
0 \\
\left(A \pi+N_{5} \alpha B\right)
\end{array}\right],
\end{aligned}
$$

where overdot indicates time derivatives.

In the above equations $A$ is proportional to the intensity of convective motion, $B$ is proportional to the intensity of microrotation and $C$ is proportional to the temperature difference between ascending and descending currents. Sign of $A$ and $C$ denote that warm fluid is rising and cold fluid is descending. The variable $D$ is proportional to the distortion of vertical temperature profile, a positive value indicating the strongest gradient occuring near boundaries.

The fourth-order Lorenz system (28) is uniformly bounded in time and possesses many properties of the full system. Also, the phase-space volume contracts at a uniform rate given by

$$
\begin{aligned}
\frac{\partial \dot{A}}{\partial A} & +\frac{\partial \dot{B}}{\partial B}+\frac{\partial \dot{C}}{\partial C}+\frac{\partial \dot{D}}{\partial D} \\
& =-\left\{\frac{\operatorname{Pr}}{\lambda}\left[D a \delta^{2}+\left(N_{1}^{\prime}+N_{1}\right) \delta^{4}\right]+\frac{P r}{N_{2} \lambda}\left(2 N_{1}+N_{3} \delta^{2}\right)+\delta^{2}+4 \pi^{2}\right\}
\end{aligned}
$$

which is always negative and therefore the system is bounded and dissipative. As a result the trajectories are attracted to a fixed point, a limit cycle or perhaps, a strange attractor. An analytical solution to the full problem in Eq. (28) is prohibitive. However, in the steady state a closed form solution can be obtained. This is demonstrated below. 


\section{Steady-state solution}

Assuming the amplitudes are steady, we set $\dot{A}=\dot{B}=\dot{C}=\dot{D}=0$ and get the following set of equations as the deterministic set for the steady amplitudes:

$$
\begin{aligned}
& A\left[D a \delta^{2}+\left(N_{1}^{\prime}+N_{1}\right) \delta^{4}\right]+N_{1} \delta_{1}^{2} B+R_{\mathrm{m}} C \pi=0, \\
& \left(2 N_{1}+N_{3} \delta^{2}\right) B+N_{1} \delta^{2} A(t)=0, \\
& \pi \alpha(1+\pi D)\left(A+N_{5} B\right)+\delta^{2} C=0, \\
& -4 \pi^{2} D+\frac{\pi^{2} \alpha C}{2}\left(A+N_{5} B\right)=0 .
\end{aligned}
$$

The steady solutions are very useful because they predict that a finite amplitude solution to the system is possible for subcritical values of the Rayleigh number and that the minimum values of $R_{\mathrm{m}}$ for which a steady solution is possible lies below the critical values for instability to either a marginal state or an overstable infinitesimal perturbation.

Writing all amplitudes in terms of $A$, we get

$$
\begin{aligned}
& B=-\frac{N_{1} \delta^{2} A}{\left(N_{3} \delta^{2}+2 N_{1}\right)}, \\
& C=\frac{A\left\{N_{1}^{2} \delta^{4}-\left[D a \delta^{2}+\left(N_{1}^{\prime}+N_{1}\right) \delta^{4}\right]\left(N_{3} \delta^{2}+2 N_{1}\right)\right\}}{R_{\mathrm{m}} \pi \alpha\left(N_{3} \delta^{2}+2 N_{1}\right)}
\end{aligned}
$$

and

$$
D=\frac{A^{2}}{8}\left\{N_{1}^{2} \delta^{4}-\left[D a \delta^{2}+\left(N_{1}^{\prime}+N_{1}\right) \delta^{4}\right]\left(N_{3} \delta^{2}+2 N_{1}\right)\right\}\left\{\left(N_{3} \delta^{2}+2 N_{1}\right)-N_{1} N_{5} \delta^{2}\right\} .
$$

Substituting Eqs. (34)-(36) into (30), we get

$$
\frac{A^{2}}{8}=\frac{\left\{R_{\mathrm{m}} \pi^{2} \alpha^{2}\left(P_{1}\right)-\delta^{2}\left(P_{2}-P_{3}\right)\left(N_{3} \delta^{2}+2 N_{1}\right)^{2}\right\}}{\pi^{2} \alpha^{2}\left(P_{2}-P_{3}\right) P_{4}^{2}},
$$

where

$$
\begin{aligned}
& P_{1}=N_{1} N_{5} \delta^{2}-\left(N_{3} \delta^{2}+2 N_{1}\right), \\
& P_{2}=N_{1}^{2} \delta^{4} \\
& P_{3}=\left(N_{3} \delta^{2}+2 N_{1}\right)\left[\mathrm{Da} \delta^{2}+\left(N_{1}+N_{1}^{\prime}\right) \delta^{4}\right], \\
& P_{4}=\left(N_{3} \delta^{2}+2 N_{1}\right)-N_{1} N_{5} \delta^{2} .
\end{aligned}
$$

The solution $A=0$ corresponds to pure conduction, which we know to be a possible solution though it is unstable when $R$ is sufficiently large. 


\section{Heat transport}

In the study of convection, the onset of convection as the Rayleigh number is increased is more readily detected by its effect on the heat transfer. In the quiescent state, the heat transfer is due to conduction alone. Hence, if $H$ is the rate of heat transfer per unit area, then we have

$$
H=-\chi_{\mathrm{e}}\left[\left\langle\frac{\partial T_{\text {Total }}}{\partial z}\right\rangle\right]_{z=0},
$$

where the angular brackets \langle\rangle correspond to a horizontal average and

$$
T_{\text {Total }}=\left(T_{0}-\Delta T \frac{z}{d}\right)+T(x, z, t) .
$$

The first term on the RHS of Eq. (39) is the temperature distribution of conduction state prevalent before convection sets in. Then, by using the last relation of (27) in Eq. (39) and using the resultant equation in Eq. (38), we get

$$
\frac{H}{\frac{\chi_{\mathrm{c}} \Delta T}{d}}=1-2 \pi D .
$$

The second term on the RHS of Eq. (40) represents the heat which enters the bottom by conduction and carried to the top by both conduction and convection and hence heat transfer increases above that by conduction alone. This process can be explained physically by a relationship between the driving temperature difference $\Delta T$ and the heat transport. Expression (40) is nothing but the non-dimensional heat transport quantity namely the Nusselt number, $N u$, which is a measure of the ratio of the heat transported across any layer to the heat which could be transported by conduction alone. Thus

$$
N u=1-2 \pi D(t) .
$$

The effects of micropolar parameters and porous parameter on heat transport/streamlines are studied and the results are discussed in the next section.

\section{Results and discussions}

In the paper we study both linear and non-linear convection in a micropolar fluid occupying a porous medium. The analyses are restricted to steady state as it is the preferred mode of convection for micropolar fluids. To study the linear theory the eigenvalue, $R_{\mathrm{mc}}$ is obtained and in the non-linear theory the heat transfer quantity, the Nusselt number, $N u$, is obtained as a function of micropolar and porous medium parameters. The linear theory results are reported in Tables $1-5$ and non-linear theory results are reported in the form of graphs in Figs. 1-5. Further the stream lines are also drawn to depict cell pattern.

Before we delve into a discussion of the results depicted by figures and tables we first make some comments on the parameters $N_{1}, N_{3}$ and $N_{5}$ arising due to the suspended particles. Assuming the Clausius-Duhem inequality, Eringen [3] presented certain thermodynamic restrictions which lead to non-negativeness of $N_{1}, N_{3}$ and $N_{5}$. For $G=0$, i.e., $N_{1}=0$ it is clear that Eq. (10) becomes independent of $G$, i.e. it is uncoupled. As $G \rightarrow \infty$, we see that $N_{1 \rightarrow 1}$ and $N_{3 \rightarrow}$. This is the Stokesian description of suspension. Thus it is obvious that couple stress comes into play at small values of $N_{3}$. This supports the contention that $N_{1 \in[}[0,1]$ and that $N_{3}$ is a small positive real number. The parameter $N_{5}$ has to be finite because the increasing of concentration has to practically stop somewhere and hence it has to be a positive, finite real number. 


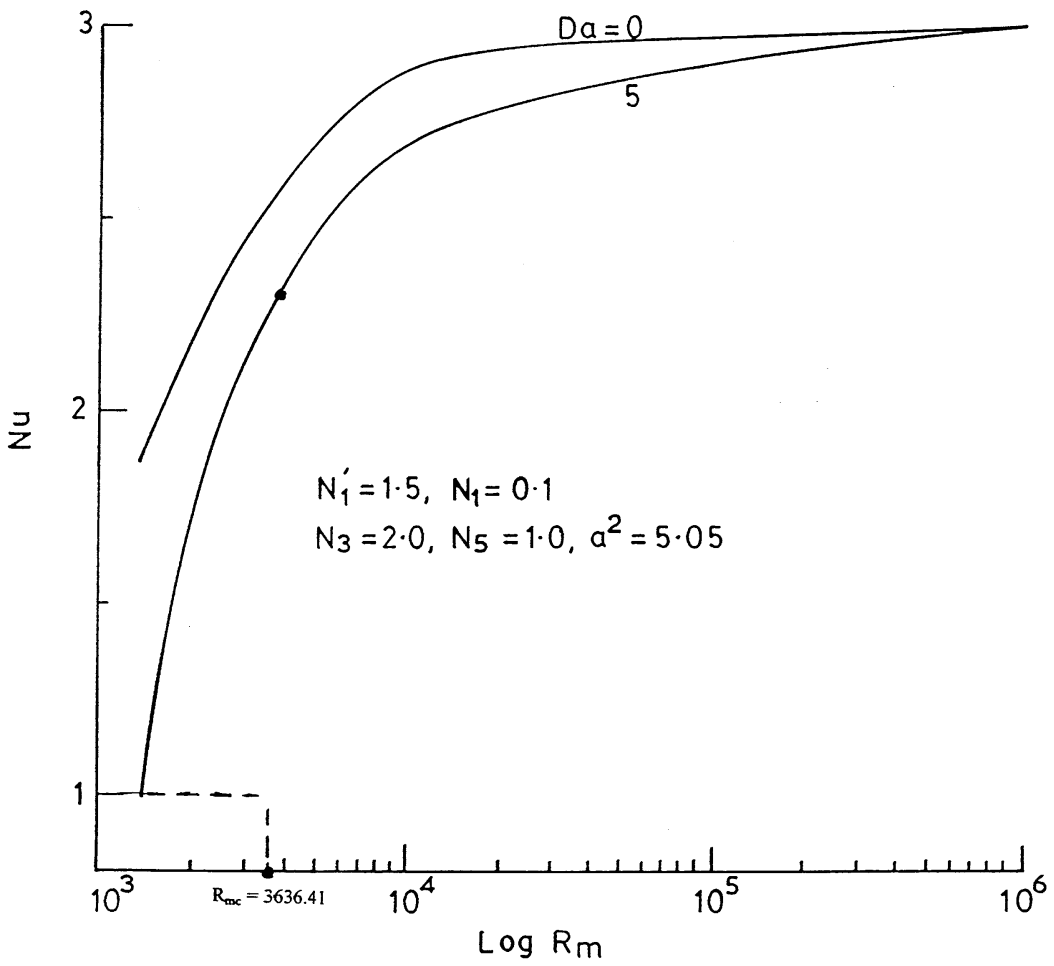

Fig. 1. Plot of Nusselt number $N u$ vs. Rayleigh number $R_{\mathrm{m}}$.

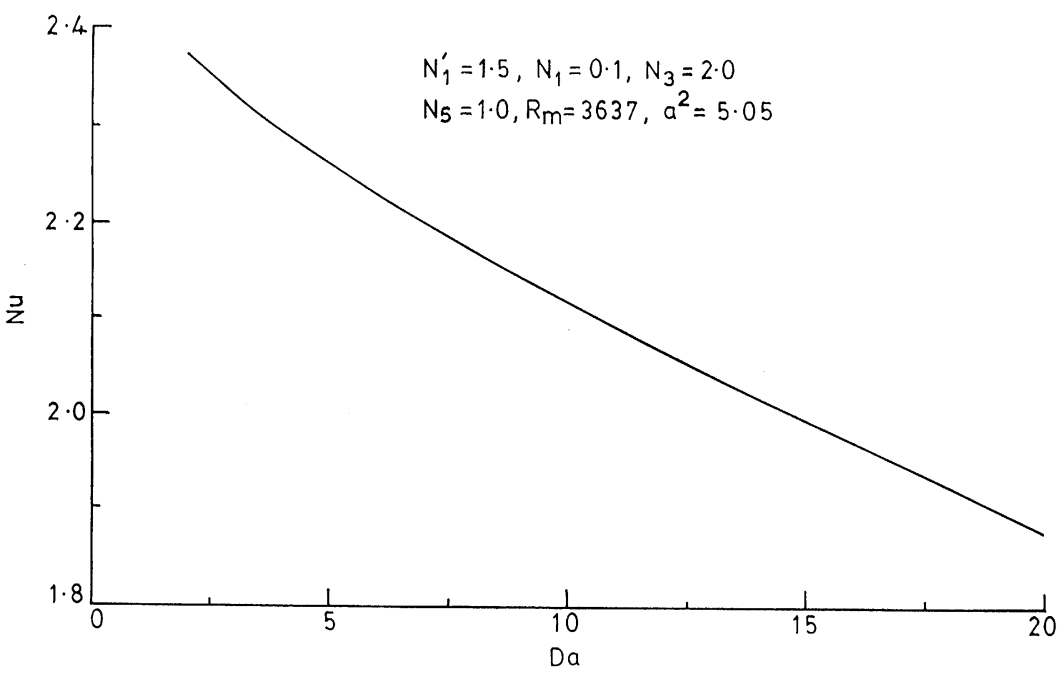

Fig. 2. Plot of $N u$ vs. inverse Darcy number $D a$. 


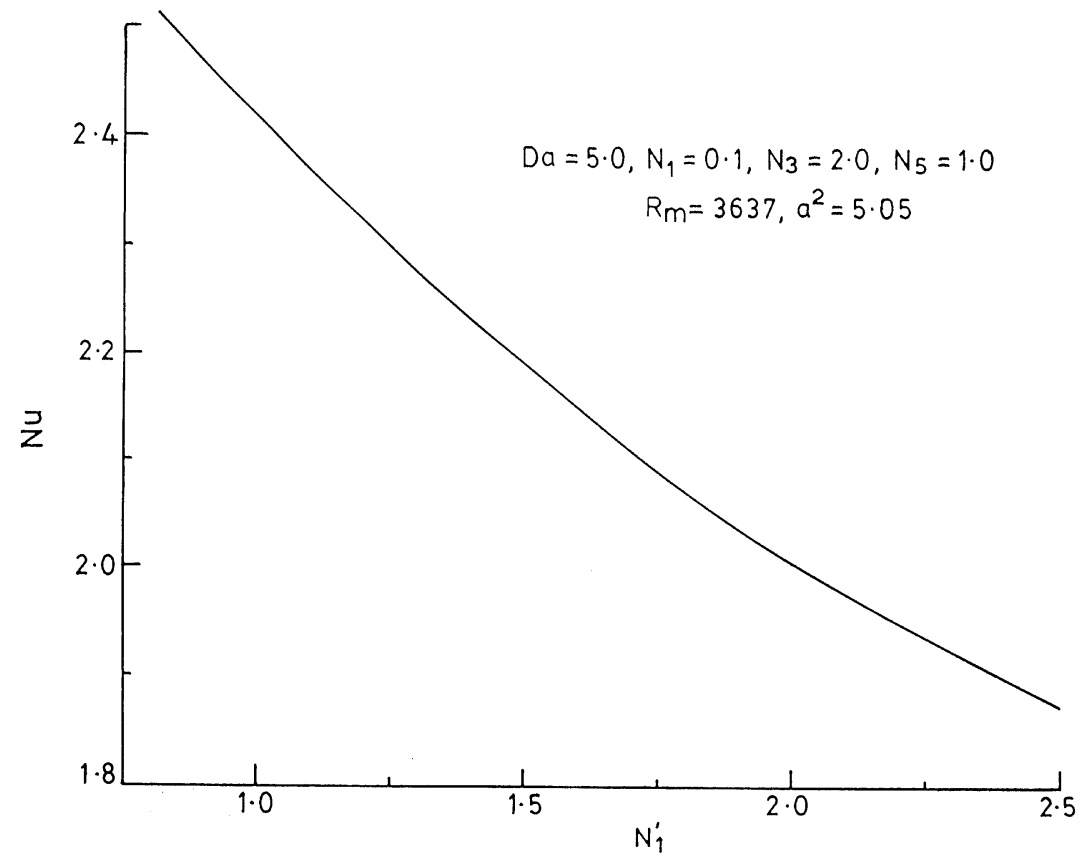

Fig. 3. Plot of $N u$ vs. modified coupling parameter $N_{1}^{\prime}$.

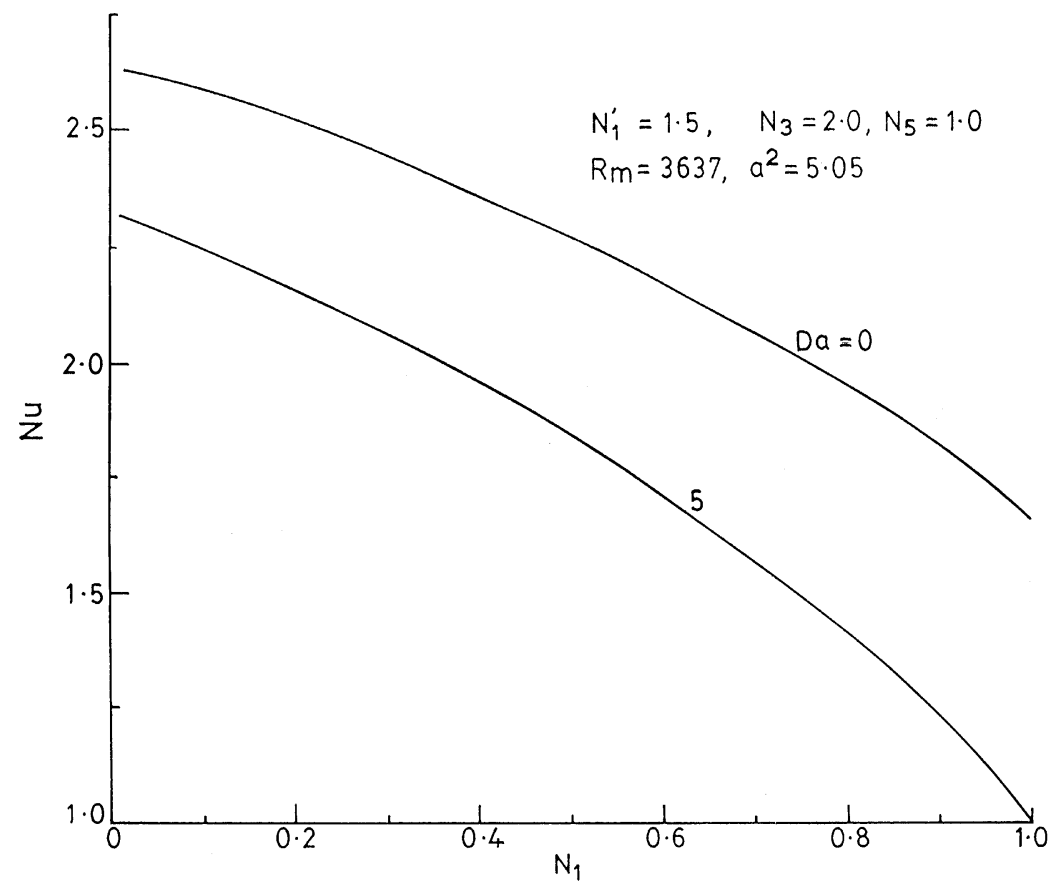

Fig. 4. Plot of $N u$ vs. coupling parameter $N_{1}$. 


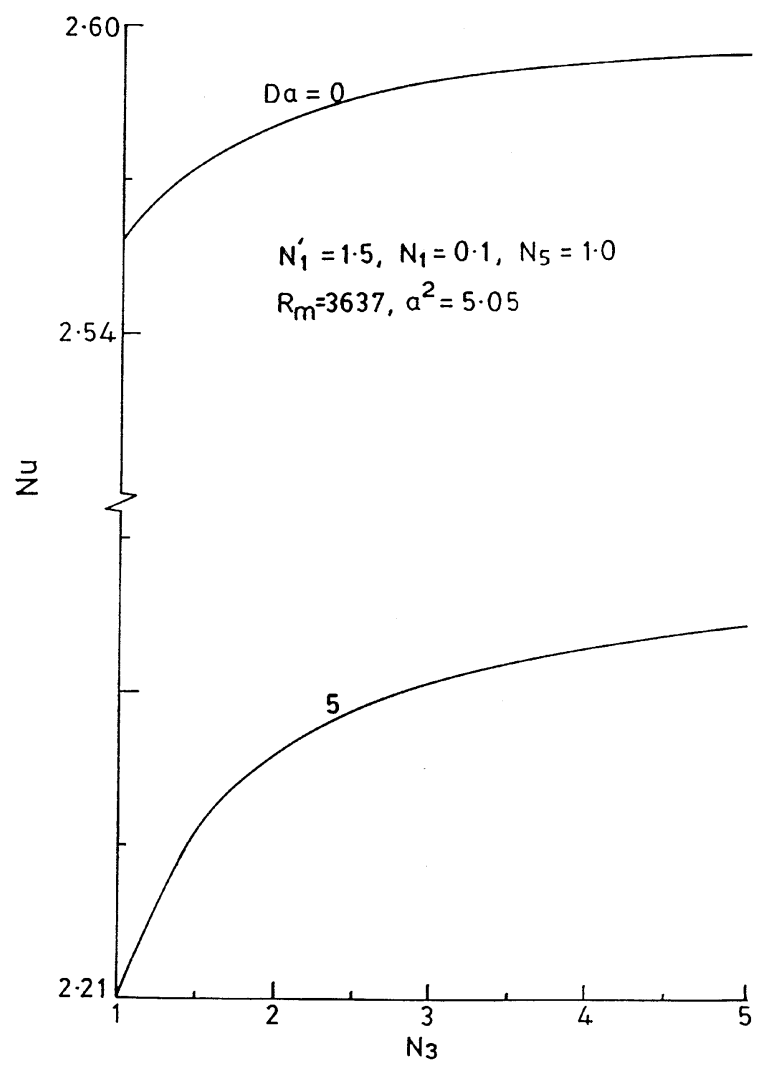

Fig. 5. Plot of $N u$ vs. couple stress parameter $N_{3}$.

From Table 1 we observe that the qualitative effect of inverse Darcy number, $D a$, on the onset of convection is the same for the both Newtonian and micropolar fluids. The effect of increase in porous packing is to delay the onset of convection.

The effect of modified coupling parameter, $N_{1}^{\prime}$, on the onset of convection is illustrated in Table 2. Increase in the effective viscosity of the porous medium is to increase the critical effective micropolar Rayleigh number, $R_{\mathrm{mc}}$.

Tables 1 and 2 indicate that the effect of parameters of porous medium is independent of type of fluid. However difference in Rayleigh number between viscous and porous case is more (significant) for micropolar fluids compared to classical fluids. Hence the Darcy friction proves to be an efficient measure of controlling convection in micropolar fluids.

Computation of $a_{\mathrm{c}}^{2}$ for different values of $D a$ and $N_{1}^{\prime}$ reveals that the effect of increase in the porous packing is to decrease the horizontal scale of cells.

Results indicated in Tables 3-5 reveal that the porous medium does not alter the effect of various micropolar parameters on $R_{\mathrm{mc}}$. From these tables we find $R_{\mathrm{mc}}$ and $a_{\mathrm{c}}^{2}$ increase with

(i) increase in $N_{1}, N_{1}^{\prime}$ and $N_{5}$ and

(ii) decrease in $N_{3}$.

These results are true for known physical reasons (see $[11,12])$. 


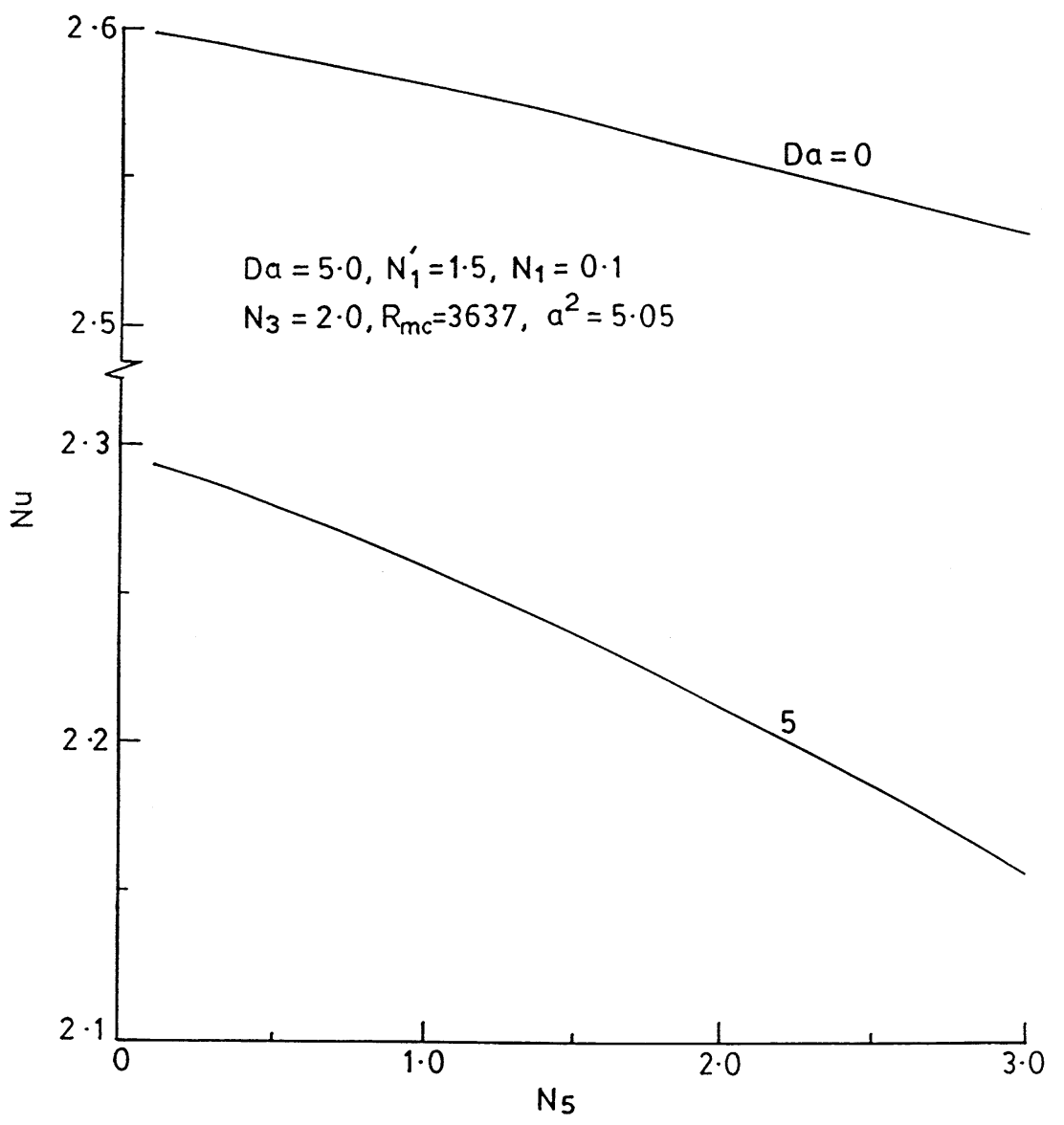

Fig. 6. Plot of $N u$ vs. micropolar heat conduction parameter $N_{5}$.

Fig. 1 depicts the effect of effective micropolar Rayleigh number, $R_{\mathrm{m}}$ on $N u$. As $R_{\mathrm{m}}$ increases, $N u$ increases exponentially and attains a maximum value of 3 . From the $N u-R_{\mathrm{m}}$ plane we observe that for any particular value of $R_{\mathrm{m}}$

$$
(N u)_{\text {porous }}<(N u)_{\text {viscous }} \text {. }
$$

The most important result from the present non-linear study can be extracted from Fig. 1. For the range of parameters reported in Fig. 1, the critical Rayleigh number and wave number from linear theory are $R_{\mathrm{mc}}=3636.41$ and $a_{\mathrm{c}}=2.247$. We note from Fig. 1 that for values of Rayleigh number less than $R_{\mathrm{mc}}$ the Nusselt number has a super-linear value indicating 'subcritical' instability. In the absence of non-linear terms the $N u-R_{\mathrm{m}}$ would go along the dotted line upto $R_{\mathrm{m}}=R_{\mathrm{mc}}$.

Effects of parameters arising due to porous medium, namely, the Inverse Darcy number, $D a$ and the modified coupling parameter, $N_{1}^{\prime}$ on $N u$ are shown in Figs. 2 and 3, respectively. The linear theory results reveal that effect of $D a$ and $N_{1}^{\prime}$ is to delay the onset of convection (see Tables 1 and 2). Hence the heat transfer across fluid layers is less, as the convective motion is restricted by these parameters and heat transfer is dominated by conduction at higher values of $D a$ and $N_{1}^{\prime}$. 

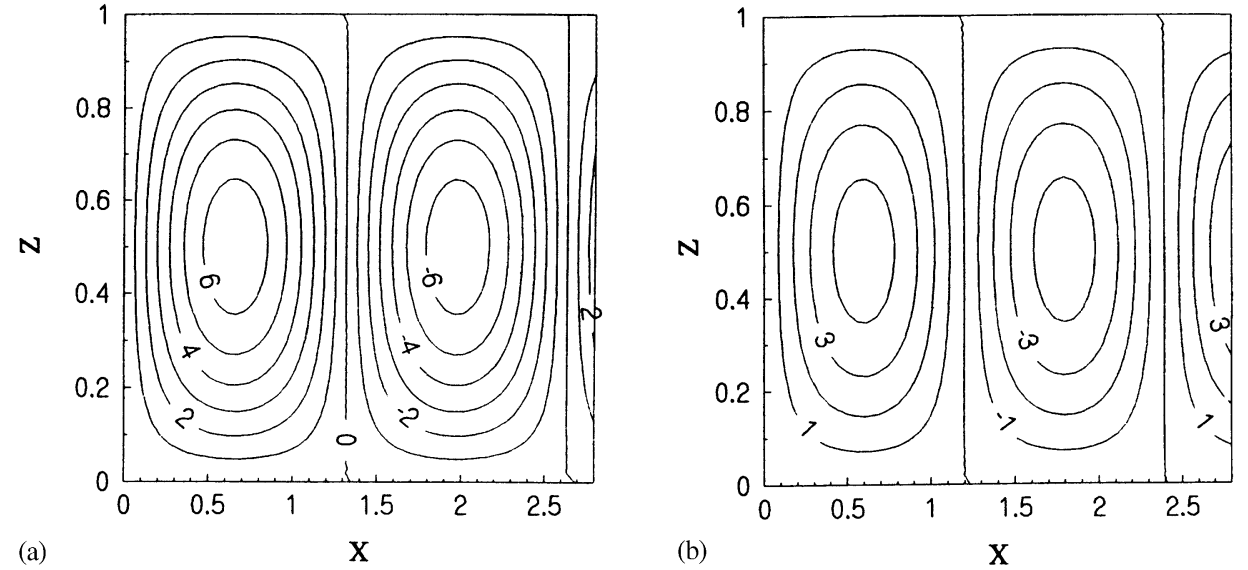

Fig. 7. Stream function profiles for different $D a$ : (a) $D a=5.0$, (b) $D a=20.0$, and $N_{1}^{\prime}=1.5, N_{1}=0.1, N_{3}=2.0, N_{5}=1.0 ; R_{\mathrm{m}}=3637, a^{2}=5.05$.
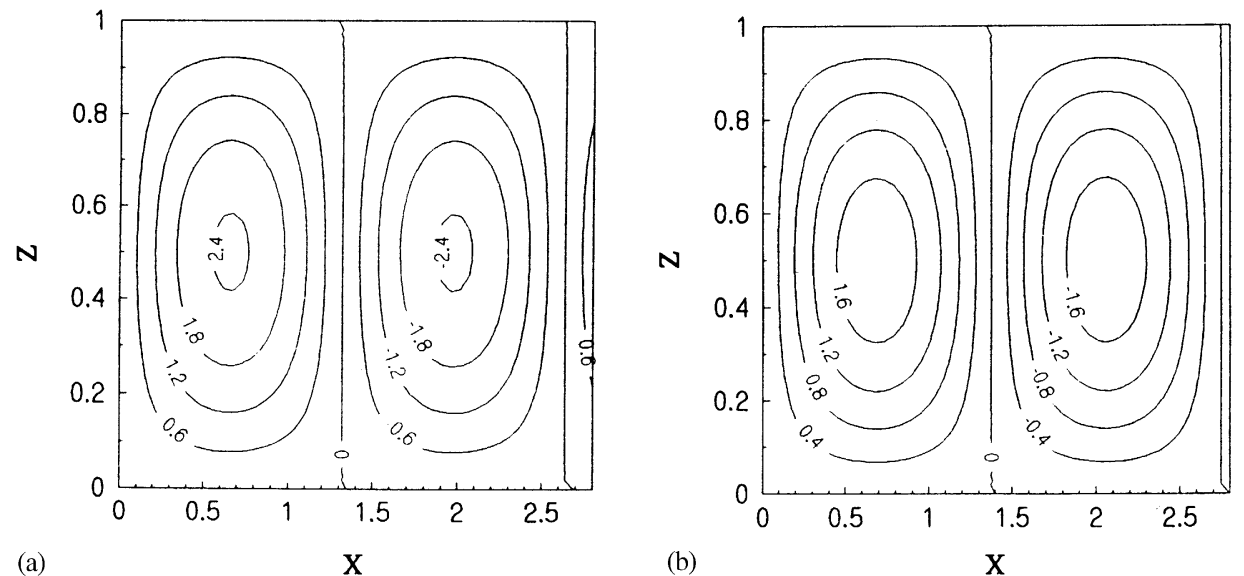

Fig. 8. Stream function profiles for different $N_{1}^{\prime}$ : (a) $N_{1}=1.5$, (b) $N_{1}=2.5$, and $D a=5.0, N_{1}^{\prime}=1.5, N_{1}=0.1, N_{3}=2.0, N_{5}=1.0 ; R_{\mathrm{m}}=3637$, $a^{2}=5.05$.

Fig. 4 illustrates the effect of coupling parameter, $N_{1}$ on $N u$. Clearly $N u$ decreases with increase in $N_{1}$. Increase in concentration of microelements (suspended particles) leads to an increase in the critical value of $R_{\mathrm{m}}$ (see Table 3 ) and hence to a decrease in heat transfer across the fluid layers.

The effect of couple stress parameter, $N_{3}$, on $N u$ is demonstrated in Fig. 5. The figure indicates that as $N_{3}$ increases the heat transport increases and levels off to the Newtonian value, both in viscous and porous cases. We may infer from this figure that micropolar fluids characterised by small values of $N_{3}$ allow less heat transfer compared to Newtonian fluids.

Fig. 6 is the plot of micropolar heat conduction parameter, $N_{5}$, versus $N u$. In the previous section we noted that effect of suspended particles is to deduct from the velocity (see Eq. (4)) and this results in reduced heat transfer by convection from the bottom to top. The figure essentially confirms the reduced heat transfer in fluids with suspended particles compared to that of fluids without suspended particles.

Figs. 7-11 show the contour plot of stream lines. The findings from the linear theory on the various parameters influence on $a_{\mathrm{c}}$ are reiterated by these figures. Increase in $N_{1}$ is to decrease $a_{\mathrm{c}}$ and thereby 

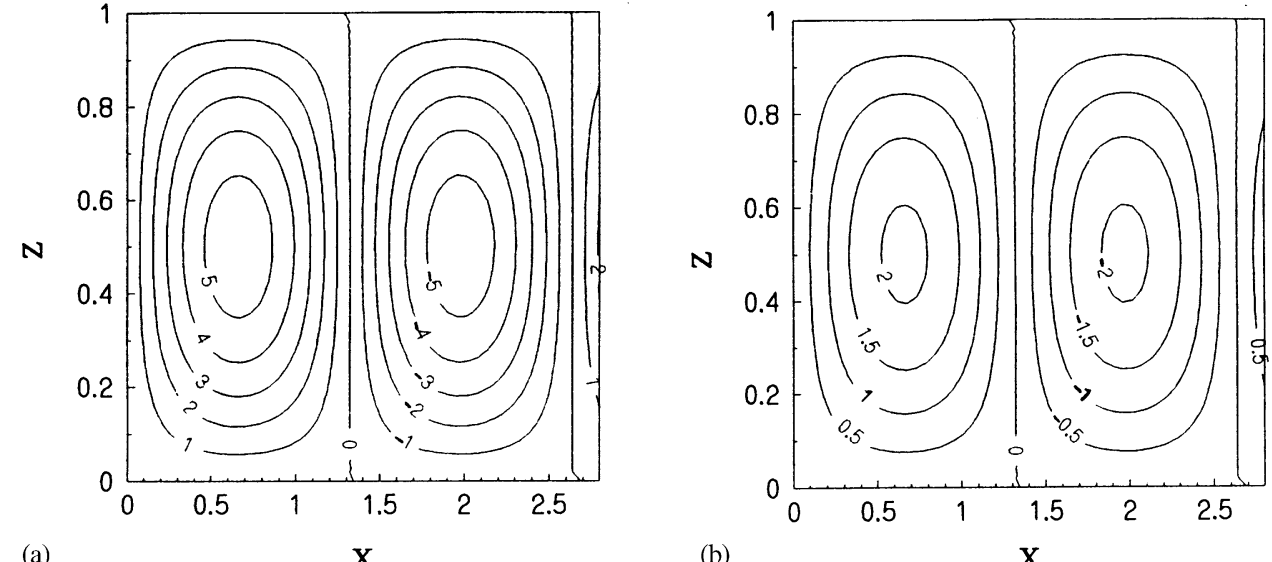

(a)

$\mathbf{X}$

(b)

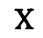

Fig. 9. Stream function profiles for different $N_{1}$ : (a) $N_{1}=0.5$, (b) $N_{1}=1.0$, and $D a=5.0, N_{1}^{\prime}=1.5, N_{3}=2.0, N_{5}=1.0 ; R_{\mathrm{m}}=3637, a^{2}=5.05$.

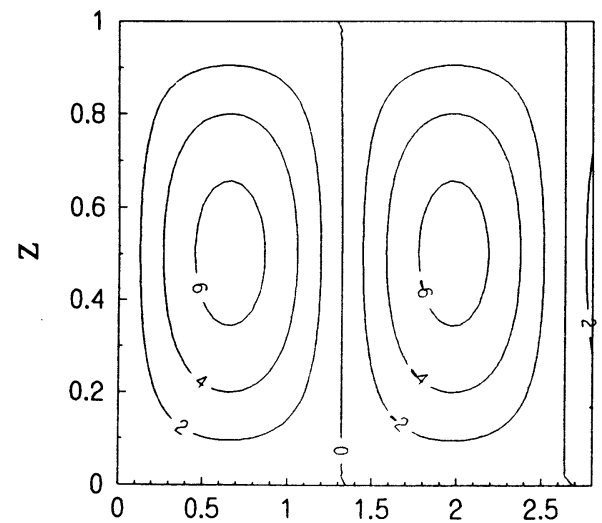

(a)

$\mathrm{X}$

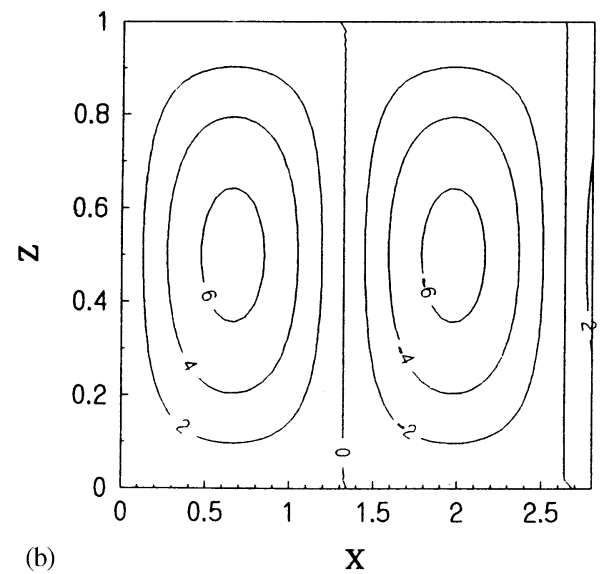

Fig. 10. Stream function profiles for different $N_{3} / N_{5}$ : (a) $N_{3}=0.5, N_{5}=0.5$; (b) $N_{3}=3.0, N_{5}=5.0$, and $D a=5.0, N_{1}^{\prime}=1.5, N_{1}=0.1$, $N_{5}=1.0 / N_{3}=2.0 ; R_{\mathrm{m}}=3637, a^{2}=5.05$.

reduced cell size. The streamlines are virtually unaffected by $N_{3}$ and $N_{5}$ but are significantly affected by $D a$, $N_{1}$ and $N_{1}^{\prime}$. Fig. 11 shows the decreasing cell-size with increase in $D a$ and $N_{1}^{\prime}$.

\section{Acknowledgements}

The work was supported by the UGC-DSA program at the Department of Mathematics, Bangalore University, India. The second author (C.V. Srikrishna) would like to acknowledge the support of the college for encouragement to do the research work. The authors are grateful to Prof. K.R. Rajagopal for calling our focused attention to many aspects of the considered problem which led us to revise our paper into a more comprehensive version. 

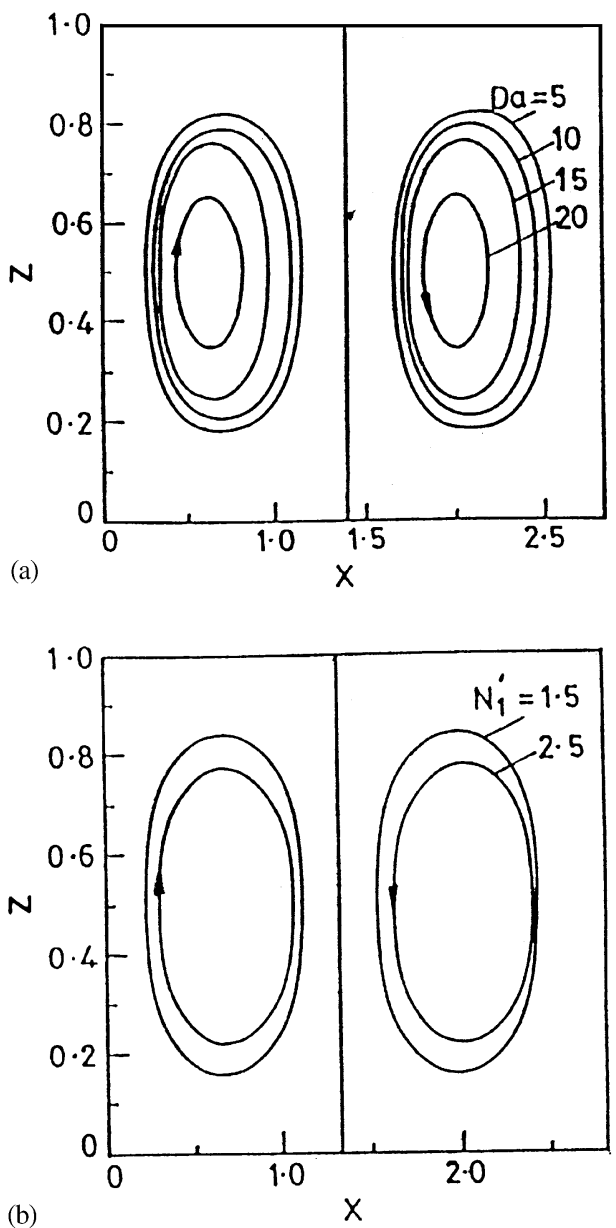

(b)

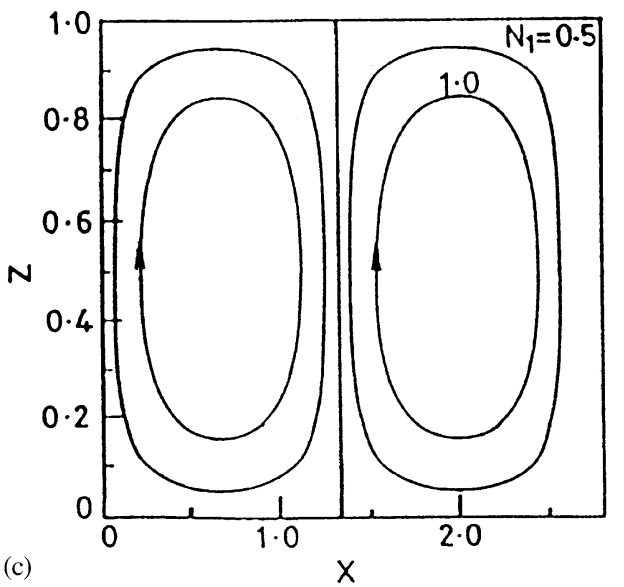

Fig. 11. Stream function profiles for (a) $\psi=4, N_{1}^{\prime}=1.5, N_{1}=0.1, N_{3}=2.0, N_{5}=1.0$; (b) $\psi=1.2, D a=5, N_{1}=0, N_{3}=2.0, N_{5}=1.0$; (c) $\psi=1.0, D a=5, N_{1}^{\prime}=1.5, N_{3}=2.0, N_{5}=1.0$. 


\section{References}

[1] H. Power, Bio-fluid Mechanics, Series on Advances in Fluid Mechanics, WIT Press, UK, 1997.

[2] G. Lukaszewicz, Micropolar Fluids: Theory and Applications, Birkhauser, Boston, MA, USA, 1998.

[3] A.C. Eringen, Theory of thermomicrofluids, J. Math. Anal. Appl. 38 (1972) 480-496.

[4] A.C. Eringen, Microcontinum Field Theories, Springer, Berlin, 1999.

[5] N.P. Migoun, Experimental method of determining parameters characterizing the microstructure of micropolar fluids, Int. J. Eng. Sci. 41 (2) (1981) 220-224.

[6] A.B. Datta, V.U.K. Sastry, Thermal instability of a horizontal layer of micropolar fluid heated from below, Int. J. Eng. Sci 14 (1976) 631-637.

[7] G. Ahmadi, Stability of a micropolar fluid layer heated from below, Int. J. Eng. Sci. 14 (1976) 81-89.

[8] G. Lebon, G. Perez-Garcia, Convective instability of a micropolar fluid layer by the method of energy, Int. J. Eng. Sci. 19 (1981) 1321-1329.

[9] B. Jena Bhattacharya, Critical effective Rayleigh number for oscillatory and non-linear convection in an isotropic thermal micropolar fluid, Int. J. Eng. Sci. 27 (1984) 13-26.

[10] Y. Qin, P.N. Kaloni, Thermal instability problem in a rotating micropolar fluid, Int. J. Eng. Sci. 30 (1992) $1117-1123$.

[11] P.G. Siddheshwar, S. Pranesh, Magneto convection in a micropolar fluid, Int. J. Eng. Sci. 36 (1998a) 1173-1181.

[12] P.G. Siddheshwar, S. Pranesh, Effect of a non-uniform basic temperature gradient on Rayleigh-Benard convection in a micropolar fluid, Int. J. Eng. Sci. 36 (1998b) 1183-1196.

[13] P.G. Siddheshwar, S. Pranesh, Effect of temperature/gravity modulation on the onset of magneto-convection in weak electrically conducting fluids with internal angular momentum, J. Math. Methods Appl. Sci. 192 (1999) 159-176.

[14] P.G. Siddheshwar, S. Pranesh, Effect of temperature/gravity modulation on the onset of magneto-convection in electrically conducting fluids with internal angular momentum, J. Math. Methods Appl. Sci. 219 (2000) 153-160.

[15] P.G. Siddheshwar, S. Pranesh, Suction-Injection effects on the onset of Rayleigh-Benard-Marangoni convection in a fluid with suspended particles, Acta Mech. 152 (1-4) (2001) 241-252.

[16] P.G. Siddheshwar, S. Pranesh, Effects of non-uniform temperature gradient and magnetic field on the onset of convection in fluids with suspended particles under microgravity conditions, Ind. J. Eng. Mater. Sci. 8 (2001) 77-83.

[17] P.G. Siddheshwar, S. Pranesh, Magnetoconvection in fluids with suspended particles under $1 \mathrm{~g}$ and mg, J. Aerospace Sci. Technol. 6 (2002) 105-114.

[18] P.G. Siddheshwar, A. Desseaux, Effects of homogeneous chemical reaction on solutal analog of Rayleigh-Benard convection in micropolar fluid, Int. J. Appl. Mech. Eng. 7 (2002) 513-525.

[19] R.C. Sharma, U. Gupta, Thermal convection in micropolar fluids in a porous medium, Int. J. Eng. Sci. 33 (1995) $1887-1892$.

[20] R.C. Sharma, Pardeep Kumar, Effect of suspended particles on thermal instability in Rivlin-Ericksen elastico-viscous fluid, Indian J. pure Appl. Math. 30 (5) (1998) 477-484.

[21] M.A. El-Hakiem, Effect of transverse magnetic field on natural convection in boundary layer flow field of micropolar fluids in a porous medium, Appl. Mech. Eng. Poland 4 (3) (1999) 508-509.

[22] L.E. Payne, B. Straughan, Critical effective Rayleigh number for oscillatory and non-linear convection in an isotropic thermal micropolar fluid, Int. J. Eng. Sci. 27 (1989) 827-836.

[23] F. Franchi, B. Straughan, Non-linear stability for thermal convection in a micropolar fluid with temperature dependent viscosity, Int. J. Eng. Sci. 30 (1992) 1349-1360.

[24] K. Vajravelu, R.N. Mohapatra, E. Soewono, Second-order non-linear system arising in convection flow of micropolar fluid, Non-Linear Anal. Theory, Methods Applications 22 (1994) 1409-1421.

[25] K.R. Rajagopal, M. Ruzicka, A.R. Srinivasa, On the Oberbeck-Boussinesq Approximation, Math. Model Methods Appl. Sci. 6 (1996) 1157-1167.

[26] K.R. Rajagopal, Tao, Mechanics of Mixtures, World Scientific Press, Singapore, 1995.

[27] K. Vafai, Handbook of Porous Media, Marcel Dekker, New York, 2000.

[28] N. Rudraiah, P.G. Siddheshwar, T. Masuoka, Nonlinear convection in porous media: a review, Int. J. Porous Media (2002), in press.

[29] S. Chandrasekhar, Hydrodynamic and Hydromagnetic Stability, Oxford University Press, London, 1961.

[30] P.G. Siddheshwar, S. Pranesh, A weak nonlinear stability analysis of convection in a micropolar fluid, Int. J. Eng. Sci. (2002), submitted for publication.

[31] G. Veronis, Cellular convection with finite amplitude in a rotating fluid, J. Fluid Mech. 5 (1959) 401-435. 